Mitophagy Balance in Various Cell Subsets in Patients with ANCA-Associated Vasculitis and Correlation with the Presence of Anti-Neutrophil Cytoplasmic Antibodies

Aggelos Banos, Konstantinos Thomas, Panagiotis Garantziotis, Nikos Malissovas, Anastasia Filia, Antigoni Pieta, Dionysios Nikolopoulos, Savina Moustafa, Alexandros Panagiotopoulos, Dimitrios T. Boumpas, Dimitrios Vassilopoulos

Mediterr J Rheumatol 2020;31(3):366-8

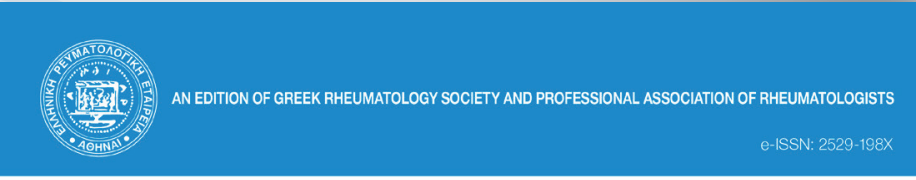

MEDITERRANEAN JOURNAL OF RHEUMATOLOGY
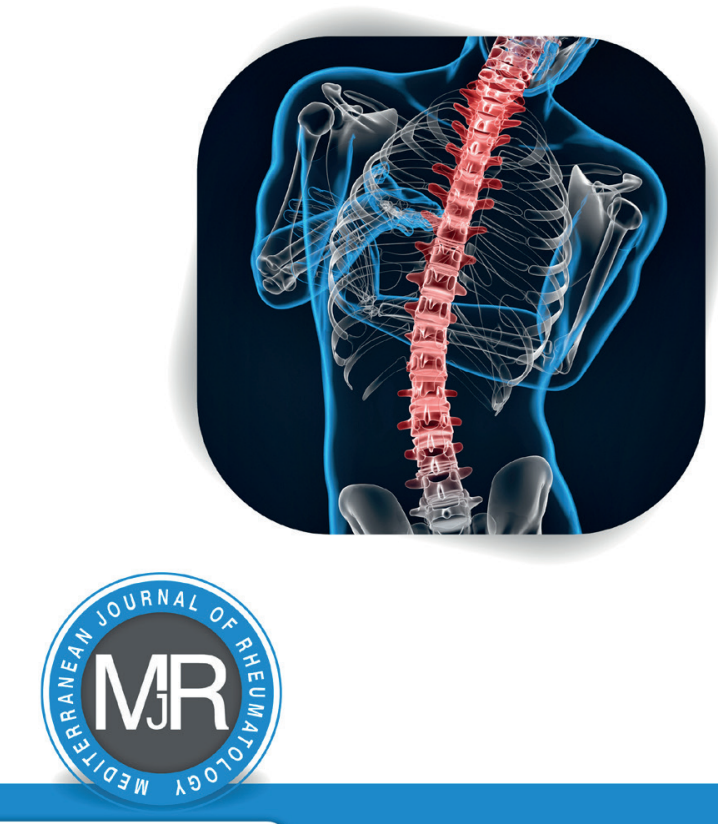

http://www.mirheum.
@MJR journal September 2020 | Volume 31 | issue 3 


\section{Mitophagy Balance in Various Cell Subsets in Patients with ANCA-Associated Vasculitis and Correlation with the Presence of Anti-Neutrophil Cytoplasmic Antibodies}

\section{Aggelos Banos $^{1}$ (D), Konstantinos Thomas ${ }^{2}$ (D), Panagiotis Garantziotis ${ }^{1,3}$, Nikos Malissovas', Anastasia Filia ${ }^{1}$ (iD), Antigoni Pieta ${ }^{3}$ (D), Dionysios Nikolopoulos ${ }^{3}$, Savina Moustafa ${ }^{2}$, Alexandros Panagiotopoulos ${ }^{2}$, Dimitrios T. Boumpas ${ }^{1,3}$ (iD), Dimitrios Vassilopoulos ${ }^{2}$ (iD)}

'Biomedical Research Foundation of the Academy of Athens, Greece, ${ }^{2}$ Joint Rheumatology Program, National and Kapodistrian University of Athens, School of Medicine, Clinical Immunology-Rheumatology Unit, $2^{\text {nd }}$ Department of Medicine and Laboratory, Athens, Greece, ${ }^{3} \mathrm{~J}$ oint Rheumatology Program, National and Kapodistrian University of Athens, School of Medicine-Clinical Immunology-Rheumatology Unit, 4th Department of Medicine, Athens, Greece

\section{ABSTRACT}

ANCA-associated vasculitides (AAVs) are characterised by heterogeneous molecular and pathophysiological traits, causing ambiguous differential diagnosis and taxonomy. Response to therapy has proven far from successful, contributing to high mortality. Transcriptome analysis of different vasculitis subtypes adds new leads in elucidating mechanisms of disease and the role of specific cell subsets to them. Recent findings have shown that mitophagy is a procedure whose imbalance could lead to immune dysregulation with certain involvement to autoimmunity. Inflammatory response related mitophagy is yet to be described in AAVs. We here describe a research protocol to investigate mitophagy in monocytes, neutrophils, and T cells in AAV patients, and the relationship of disturbed mitophagy with ANCA seropositivity.

Mediterr J Rheumatol 2020;31(3):366-8

https://doi.org/10.31138/mjr.31.3.366

Article Submitted: 3 Mar 2020; Revised Form: 3 Jun 2020; Article Accepted: 20 Jun 2020; Available Online: 4 Jul 2020

Keywords: vasculitis, autoimmunity, transcriptomics, mitophagy

\section{Corresponding Author:}

Dimitrios Vassilopoulos, MD

Professor of Medicine - Rheumatology

Joint Rheumatology Program

Clinical Immunology-Rheumatology Unit

$2^{\text {nd }}$ Department of Medicine and Laboratory

National and Kapodistrian University of

Athens

School of Medicine

Hippokration General Hospital

114 Vass. Sophias Ave.,

11527 Athens, Greece

Tel.: $+30-213-2088516$

Fax: +30-213-2088399

E-mail: dvassilop@med.uoa.gr

\section{INTRODUCTION}

ANCA (anti-neutrophil cytoplasmic antibodies) associated vasculitides (AAV) include granulomatosis with polyangiitis (GPA), microscopic polyangiitis (MPA), and eosinophilic granulomatosis with polyangiitis (EGPA) subtypes, being the most aggressive as to morbidity and mortality rates compared to other systematic vasculitides. Worldwide incidence of AAVs is approximately $26 / 10^{6}$ individuals, with higher impact at ages of 65 to 74 years. At the same time, epidemiological studies in Greece are sparse, while in Crete, the respective incidence is $19.5 / 10^{6}$ individuals, with younger patients presenting GPA, and older ones with MPA. ${ }^{1}$ Despite the intensive therapeutic approaches including corticosteroids and immunosuppressant drugs (cyclophosphamide, azathioprine, methotrexate, mycophenolate mofetil) or their combination, patients suffer from subsequent relapses and high mortality, estimated at approximately $25 \%$ in 5 years. ${ }^{2,3}$ Recently, rituximab has been approved as therapeutic solution for induction of remission and maintenance of GPA and MPA, resulting to encouraging outcomes. Independent prognostic 
factors that are associated to high mortality are advanced age, renal involvement and high activity scores (BVAS and BVAS/WG) at diagnosis. Pathogenetic mechanisms of AAVs are yet to be fully elucidated and there are no specific biomarkers discerning disease subtype, response to therapy, or disease course/relapse rate.

RNA-sequencing is a cutting-edge technology that provides tools for transcriptomic characterisation of the disease and molecular clustering of patients based on their transcriptional profile. A first study of transcriptome profiling defined a transcriptional signature of CD8 T-cell exhaustion, associated with prediction of better prognosis in AAV, along with other autoimmune diseases. ${ }^{4}$ In order to utilise this method to explain disease mechanisms and strengthen standard classification criteria, we analysed whole blood transcriptome of 42 AAV patients (with MPA, GPA or EGPA) and 11 healthy individuals. Gene ontology and enrichment analysis on differentially expressed genes between patients and healthy individuals formed a pan-vasculitis disease signature associated with deregulation of pathways related to neutrophil degranulation, immune activation, and immunometabolism among others. Distinctive gene pathways can discriminate different AAV subtypes from healthy state, giving molecular insights on differential diagnosis. Principal component analysis proved that patients with renal involvement in AAV exhibit a discrete transcriptional profile from those with SLE nephritis. Other pathways emerging from intra-disease comparisons are deregulation of cell cycle, DNA damage response, and mitophagy. We sought to study this last procedure and examine its correlation to ANCA seropositivity.

Mitochondria are fundamental effectors of immune signalling as well as rheostats of energy and ROS production. Their malfunction could result to pathology related to cytokine signalling. Mitophagy is a homeostatic mechanism that drives destroyed or redundant mitochondria to autophagy pathway, playing an important role in immune balance. 5,6,7,8,9 Recent studies in inflammatory bowel disease (IBD) and systemic lupus erythematosus (SLE) prove that mitophagy links autophagy with inflammatory response. ${ }^{10,11,12}$ The importance of the role of mitophagy in vasculitis has yet to be clarified.

\section{AIM OF THE STUDY}

Comparison of mitophagy in different cell subtypes in AAV patients and correlation with ANCA positivity. Utilisation of mitophagy pathway to assess cell phenotypes associated with disease activity and vasculitis subtype.

\section{METHODS}

Based on the initial transcriptomic data, we plan to assess mitophagy through 4 different directions.

In work package 1, validation of transcriptional profile for mitophagy genes will take place in an independent cohort of 10 patients ( $n=5$ with ANCA+ and $n=5$ with ANCA-). Recruitment criteria will include active disease (BVAS $>0$ ) and whole blood will be collected. RNA extraction will be followed by cDNA synthesis and qPCR for a specific panel of mitophagy related genes.

In work package 2, collection of different cell subtypes from the same patients (WP1) and measurement of their frequencies will be performed by either magnetic isolation or flow cytometry. Whole blood will be processed through double density Ficoll gradient, in order to isolate PBMCs and neutrophils. Flow cytometry will be exploited to assess the frequencies and purity of monocytes/macrophages (CD14, CD16), CD8 ${ }^{+} \mathrm{T}$ cells and neutrophils (CD66b, CD15). The latter neutrophilic population will be collected through FACS (fluorescence-activated cell sorting) while monocytes and CD8 T cells will be isolated through specific magnetic columns in high purity.

In work package 3, levels of expression of mitophagy genes will be measured in monocytes/macrophages, neutrophils and CD8 T cells in AAV patients. A fraction of the isolated cells from the previous work package will be processed for RNA extraction and cDNA synthesis. A selected panel of mitophagy genes will be assessed through qPCR. Results will lead to mechanistic insights of mitophagy mediated immune dysregulation.

In work package 4, relative weight of mitochondria and mitophagy procedure will be assessed in cell subsets of AAV patients, isolated previously in WP2. In particular, we plan to stain monocytes, neutrophils and CD8 T cells with MitoTracker ${ }^{\mathrm{TM}}$ Green measuring the cumulative cellular mitochondrial mass, through flow cytometry. The same cell subsets will be stained with $\mathrm{JC}-1$ assay (MitoProbe ${ }^{\mathrm{TM}}$ JC-1 Assay Kit), measuring fluctuations of membrane potential in mitochondria through flow cytometry in order to extract information about membrane functionality and relative number of functional mitochondria. Consecutive simultaneous staining with MitoTracker ${ }^{\mathrm{TM}}$ Green and LC3 will be assessed through confocal microscopy. This experiment will visualize the engulfment of mitochondria in autophagosomes and measure their processing and destruction through autophagy. Finally, superoxide production from mitochondria will be measured through a flow cytometry-based assay.

\section{ANTICIPATED BENEFITS}

Mitophagy plays a significant role in inflammation and autoimmunity. The results of this study will elucidate the involvement of mitophagy in AAV and add mechanistic insights in the pathophysiology of the disease. Moreover, assessment of mitophagy in different cell subsets will shed light on their role as effectors in the phenotype of the disease, linking transcriptome to function.

\section{CONFLICT OF INTEREST}

The authors declare no conflict of interest. 


\section{ACKNOWLEDGEMENTS}

The study is funded by the Greek Rheumatology Society and Professional Association of Rheumatologists.

\section{REFERENCES}

1. Panagiotakis $S H$, Perysinakis GS, Kritikos $H$, Vassilopoulos $D$, Vrentzos $\mathrm{G}$, Linardakis $\mathrm{M}$, et al. The epidemiology of primary systemic vasculitides involving small vessels in Crete (southern Greece): a comparison of older versus younger adult patients. Clin Exp Rheumatol 2009 May-Jun;27(3):409-15.

2. Flossmann O, Berden A, de Groot K, Hagen C, Harper L, Heijl C, et al. Long-term patient survival in ANCA-associated vasculitis. Ann Rheum Dis 2011 Mar;70(3):488-94.

3. Walsh M, Flossmann $O$, Berden A, Westman $K$, Höglund $P$, Stegeman C, et al. Risk factors for relapse of antineutrophil cytoplasmic antibody-associated vasculitis. Arthritis Rheum 2012 Feb;64(2):542-8.

4. McKinney EF, Lee JC, Jayne DR, Lyons PA, Smith KG. T-cell exhaustion, co-stimulation and clinical outcome in autoimmunity and infection. Nature 2015 Jul 30;523(7562):612-6.

5. Lazarou M, Sliter DA, Kane LA, Sarraf SA, Wang C, Burman JL, et al. The ubiquitin kinase PINK1 recruits autophagy receptors to induce mitophagy. Nature 2015 Aug 20;524(7565):309-14.

6. Nakahira K, Haspel JA, Rathinam VA, Lee SJ, Dolinay T, Lam HC, et al. Autophagy proteins regulate innate immune responses by inhibiting the release of mitochondrial DNA mediated by the NALP3 inflammasome. Nat Immunol 2011 Mar;12(3):222-30.

7. Sliter DA, Martinez J, Hao L, Chen X, Sun N, Fischer TD, et al. Parkin and PINK1 mitigate STING-induced inflammation. Nature 2018 Sep;561(7722):258-62.

8. Van den Bossche J, Baardman J, Otto NA, van der Velden S, Neele $A E$, van den Berg SM, et al. Mitochondrial dysfunction prevents repolarization of inflammatory macrophages. Cell Rep 2016 Oct 11;17(3):684-96

9. Singh SB, Ornatowski W, Vergne I, Naylor J, Delgado M, Roberts $\mathrm{E}$, et al. Human IRGM regulates autophagy and cell-autonomous immunity functions through mitochondria. Nat Cell Biol 2010 Dec;12(12):1154-65.

10. Leishangthem BD, Sharma A, Bhatnagar A. Role of altered mitochondria functions in the pathogenesis of systemic lupus erythematosus. Lupus 2016 Mar;25(3):272-81.

11. Caielli S, Athale S, Domic B, Murat E, Chandra M, Banchereau $\mathrm{R}$, et al. Oxidized mitochondrial nucleoids released by neutrophils drive type I interferon production in human lupus. J Exp Med 2016 May 2;213(5):697-713.

12. Hui KY, Fernandez-Hernandez $\mathrm{H}$, Hu J, Schaffner $\mathrm{A}$, Pankratz $\mathrm{N}$, Hsu NY, et al. Functional variants in the LRRK2 gene confer shared effects on risk for Crohn's disease and Parkinson's disease. Sci Transl Med 2018 Jan 10;10(423):eaai7795. 